\title{
A Fast Method for Measuring Kinematic Parameters of Experimental Vehicle Based on Machine Vision
}

\author{
Zi Liang and Tao Wang \\ Armored Force Academy, No 21, Dujiakan, Fengtai District, Beijing, China
}

\begin{abstract}
The vehicle kinematic parameter is important evaluation index of vehicle performance. But the measurement technique for vehicle kinematics parameters is still immature. Practical and efficient measuring system is now in badly demand. With the development of machine vision technique, more and more measurement problems can be solved better by combining the measuring task with optical system. And on this basis the accuracy and efficiency of kinematic parameter measurement can be improved. A measuring plan is presented in this paper. In order to simplify the measuring algorithm, a simple and practical marker is as the substitute of vehicle. The complex measurement system is simplified and transformed to detection and trajectory tracking of the marker. In order to reduce the amount of computation and improve the speed of detection, a motion prediction local detection theory is presented to replace the traditional background subtraction detection method. Several experiments through MATLAB are conducted to verify the feasibility and accuracy of this measuring method.
\end{abstract}

Keywords-kinematic parameters; machine vision; background subtraction; motion prediction

\section{INTRODUCTION}

With the development of mechanical automation and optical measurement system, more and more mechanical products and optical system are combined and form a variety of complex fields, such as defect detection on product line, robot automatic sorting, dimension measurement of machine parts and trajectory tracking, which are all based on machine vision.

The vehicle kinematic parameters are important evaluation index of vehicle performance, mainly include speed, acceleration, direction angle, side-slip angle and slip rate. The measurement technique of vehicle kinematics parameters is still immature. The measurement methods include direct measurement method and indirect estimation method. Using high precision instrument or sensor is of high accuracy, but it is constrained by the environment, high cost, so that it is difficult to be widely use. Although the cost of the estimation method is controllable, the measurement accuracy is difficult to guarantee because of the limitation of algorithm and instrument. Therefore, a practical and efficient kinematic parameters measurement system with both high accuracy and low cost has great prospects for development.

Vehicle position and direction detection technology based on machine vision can detect vehicle trajectory through time series images. It uses vehicle's high-precision trajectory to calculate the speed, direction angle and other parameters and calculate the kinematic parameters combined with the information from the high-precision sensor in vehicle body. As a kind of non-contact measurement method, it has the advantages of low cost, less preparation, efficient and high accuracy, at the same time, its practicability and extensiveness make it possible to measure more kinds of parameters in one platform or combine with other measuring systemto form comprehensive measurement systems.

The data for vehicle kinematic parameters calculation are mainly provided by vehicle position and direction parameters. This paper is mainly on the basis of previous research, puts forward a system for experimental vehicle's kinematic parameters measurement using a substitute marker based on machine vision, analyzes the key point of vehicle trajectory detection, recognition and measurement of the marker and presents a simple and fast method for locating and measuring the vehicle.

\section{RESEARCH STATUS OF VEHICLE DETECTION AND TRAJECTORY TRACKING}

In order to obtain the vehicle trajectory information, vehicle detection and tracking algorithms are used to process the captured images. Accurate vehicle trajectory is synthesized by the change of vehicle's position and direction information in time sequence images. There are usually the following methods:

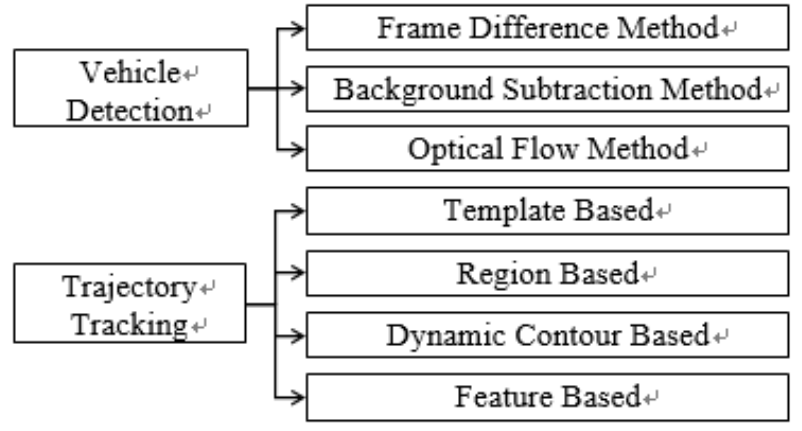

FIGURE I. MET HODS OF VEHICLE DETECT ION AND TRAJECT ORY TRACKING

Traditional methods for vehicle detection are frame difference method, background subtraction method and optical flow method, and methods for trajectory tracking are template based, region based, dynamic contour based and feature based method. Nowadays there have appeared many new methods with new theories or combined with other techniques.

\section{A. Research on Vehicle Detection}

Vehicle detection is to process the image and segment the vehicle image. Usually there are frame difference method, background subtraction method and optical flow method. The basic principle of frame difference method is using the image 
subtraction of two neighboring frames or more frames to eliminating static or slow objects and the background. [1] had research on frame difference method, [2] presented a threeframe statistics calculation method to get the coarse detection area. [3] had research on multi-frame image subtraction statistics based on Markov random field.

The basic principle of background subtraction is to obtain the object shape by comparing the captured background image and prior background image. [4] and [5] respectively had research on background subtraction method. [6] presented a selective update method, only update the region of moving object to reduce the amount of calculation. [7] set a threshold to determine the area of background as updating region.

The optical flow method mainly includes two methods: the continuous and the characteristic optical flow method. The principle is to distinguish the vehicle by calculating the velocity field of the three-dimensional motion field projected on the twodimensional image plane. [8] proposed an optical flow based middle level vision integration algorithm and got good results.

[9] built the region of interest of detection based on the transcendental model of vehicle, then used the symmetry axis to locate the exact rectangular contour of vehicle and proved it useful for vehicle detection by experiments. [10] combined shadow extraction pixel extraction and horizontal edge extraction in HSV color space to detect the region of interest. As machine learning technology is widely used in machine vision field, it is also used on vehicle detection. [11] presented a detection method which used symmetry, shadow and edge features and trained AdaBoost classifier to recognize the vehicles, and the method met the requirements of real-time and accuracy well. [12] used HOG (Histogram of Oriented Gradient) feature and SVM (Support Vector Machine) classifier to recognize the vehicles in huge scene, far distance and multiple angle circumstances. [13] proposed a vehicle hypothesis area generation method, and trained CNN (Convolutional Neural Network) to recognize the vehicle and the method worked well.

\section{B. Research on Trajectory Tracking}

Trajectory tracking is mainly to obtain the trajectory of the moving vehicle in the experimental area, and get the information of the position, velocity and orientation of the moving vehicle. It can usually be divided into four categories: model based tracking, region based tracking, dynamic contourbased tracking and feature based tracking.

Model based tracking method matches the prior vehicle model and captured image to find corresponding information of model vehicle. [14] used the pairwise geometric histogram to encode the local geometric context mark as the dynamic template to track. [15] used a deformable template to measure vehicle dimension and got good result. [16] proposed a $\mathrm{HMM}$ /MRF detection frame for robust vehicle tracking, which can distinguish the vehicle, background and shadow with high precision.

The principle of region based tracking method is using the vehicle pixel connected areas to track the trajectory of the vehicle. [17] and [18] respectively had research on region based tracking method, and the accuracy they got is good and reliable.
The principle of dynamic contour based method is to use the prior vehicle contour to track the corresponding vehicle image in each frame, and then update the contour through the subsequent frame. [19] used the real-time exchange technology to enhance the track forecast speed. [20] introduced shape deviation in integration algorithm. [21] proposed an outline initialization method based on contour growth, which combined with Kalman filter to improve the tracking accuracy.

The feature based tracking method is based on the feature information like points, lines, planes, edges, gray levels, colors, textures, shapes, etc.to match each frame image. [22] [23] [24] respectively had research on points, lines and planes to track. [25] proposed a Bayesian multiple hypothesis tracking method to track the object contour characteristics and got good results.

With the rapid development of machine learning technology, machine learning method on vehicle detection and trajectory tracking develops fast. Though artificial neural network, support vector machine and Adaboost algorithm, machine begin to gain the ability to discern and understand the image. It can solve the traditional algorithm of large computation, low real-time performance, sensitivity to environment and low accuracy. [26] had research on vehicle detection and trajectory tracking using segmental Blob and Snack Blob features through neural network classification. [27] designed a new detection algorithm using Harr wavelet features through support vector machine. [28] had research on detection using gradient analysis and AdaBoost classification algorithms.

[29] made a research on vehicle tracking by using Kalman filter and did experiments on highway while the method could detect vehicles on $120 \mathrm{~km} / \mathrm{h}$. [30] proposed a particle filter tracking algorithm based on MIL (Multiple Instance Learning) method and boosting algorithmand its performance is good. [31] presented a vehicle trajectory tracking method based on Camshift algorithm modified by Kalman filter which could track multiple vehicles at the same time. [32] used Contourlet histogram to extract the texture features of the vehicle and tracked its trajectory by Meanshift algorithm with good stability and interference immunity. [33] proposed a tracking algorithm based on AM (Appearance Model) on the basis of IPCA (Incremental Principal Component Analysis) and particle filter and improve the tracking success rate to $95.1 \% \sim 96.4 \%$.

\section{System DESIGN For Vehicle TRAJeCtory DETECTION}

In the measurement of the kinematic parameters of the model vehicle at a certain test site, there are invariant test space, single target and real-time requirement. In this situation, present a simplified method on the basis of previous research. Detect the specific marker on the top of vehicle though the time series images captured by the camera which is above the experimental area, and identify the position and direction of test vehicle to gain the kinematic parameters. This method simplifies the complexity of vehicle detection and tracking tasks, and use the detection and tracking of specific marker to replace. Further, it is the identification of specific marker and its exact position in each sequence image. On the one hand, this method and algorithm are greatly simplified; on the other hand, the computational complexity is greatly reduced, and the detection speed and real-time performance are greatly improved. 


\section{A. System Design}

The whole system consists of two parts, as shown below in Figure 2:

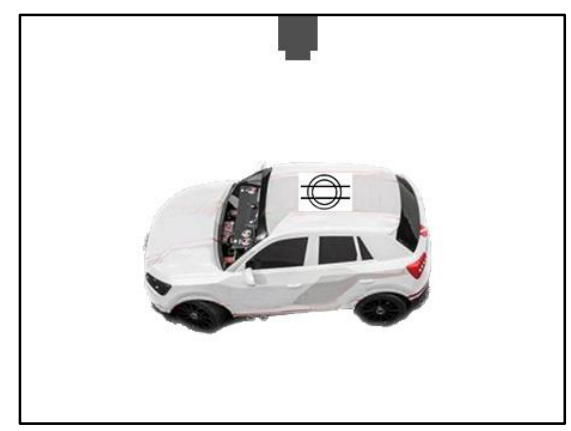

FIGURE II. MEASURING SYST EM DESIGN

The first part is a high-precision camera arranged above the experiment area, used to collect continuous time series images of moving vehicle. This part should be adjusted according to the situation of test area and the requirement of accuracy. Usually choose high-precision CCD (Charge Coupled Device) camera as the acquisition device and arrange single camera or multicamera array for different area situation and accuracy requirement. The second part is the test vehicle with a specific marker at the top. The key point of this part is the design of the marker. The marker should have clear distinction from the background, clear boundary, obvious characteristics and easy to be recognized and detected, at the same time, it must meet the precision requirement of position and direction measurement.

\section{B. Marker Design}

In this paper, in the experiment of trajectory tracking of moving vehicle in test area, a simple and practical marker is designed. The marker consists of two concentric circles and two parallel lines through the concentric circles, the overall size is as below in Figure 3:

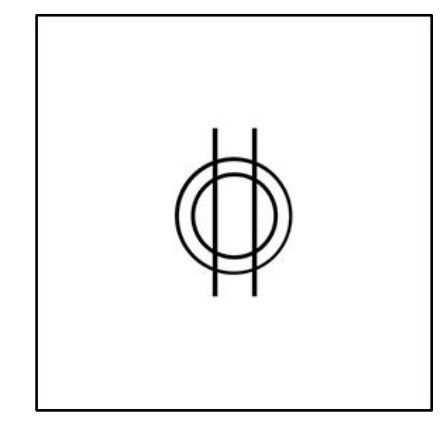

FIGURE III.MARKER DESIGN

The marker meets the requirement of high difference from background, clear boundaries, obvious characteristics and it is easy recognizable and detectable. In the measurement process, only a simple template matching can locate the marker area, and then detect the straight lines and the circles by the algorithm to achieve accurate measurement of the vehicle position and direction. Meanwhile, calculate the average center of concentric circles and average position of parallel lines can improve the accuracy of measurement.

Because the marker is used as the measurement object of vehicle position and direction, the recognition accuracy requirement is not high, so the location of the vehicle can be roughly detected only by template matching based on prior information of shape and dimension.

After the detection of the marker area, in order to gain the trajectory of the vehicle, the marker in every frame image should be detected and tracking. Due to the continuity of the vehicle movement, the location of markers in time series images must be continuous. So with recognition of the marker in the initial state, only need to directly detect lines and circles in the neighborhood of the marker position in the last frame image.

Here uses the method of Hough transformation to detect the circles and lines. Calculate the average center of two concentric circles as the position information and the average position of two parallel lines as the direction of the vehicle. The algorithm and program are conducted by MATLAB.

The average circle and line position detection result is shown below in Figure 4:

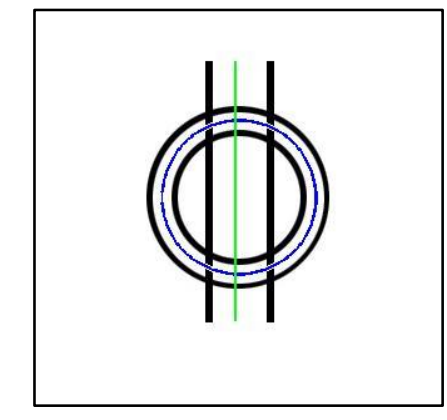

FIGURE IV.DETECTION RESULT

According to previous research and experimental verification, the relative error of position detection is $3.8 \%$ and $2.4 \%$ of angle detection [34].

\section{DETECTING AND MEASURING EXPERIMENT}

In order to detect the vehicle trajectory and measuring its accurate motion, an experiment was conducted using traditional background subtraction method to detect the trajectory and measure the motion of the vehicle. On this bas is, in order to solve the shortcoming of this method on real-time performance, a tracking detection method based on motion prediction was proposed. It uses the trajectory measuring results in last 3 frames to predict the position of vehicle in current frame, and combines with the detecting results, the average radius of the concentric circles in last frame to predict the detecting area in current frame.

The experiment uses vehicle moving video generated by computer simulation with the resolution of $1100 \times 800$ and 120 frames in all. The route of the vehicle is shown below in Figure 5 . The vehicle moves along the white line from the top right of the experiment field to the lower right. Straight and turn motion are both included. And use MATLAB to program for the two methods. The hardware and software platform consists of Windows 10 professional Intel(R) Core(TM) i7-2630QM CPU @ 2.00GHz 8G RAM+MATLAB 2014a. 


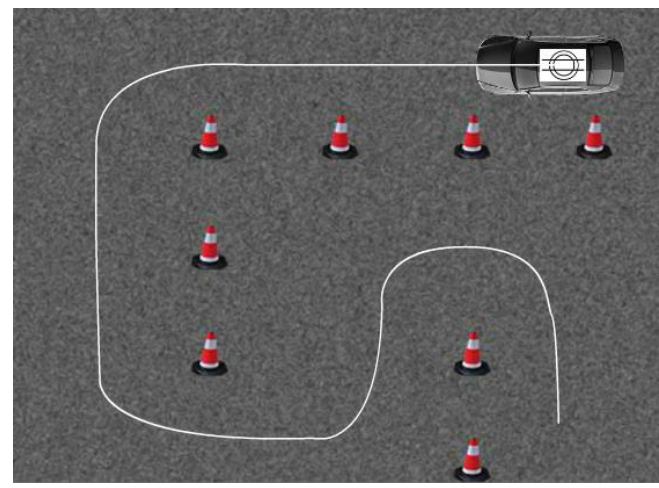

FIGURE V.EXPERIMENT ROUTE

Background subtraction is an effective method for detecting foreground images in static background images. Firstly make the model of the background. The difference between the background image pixels and its corresponding point in background model is very small, otherwise in foreground image is very large, then the foreground image can be detected by making the difference between each frame image and the background model.

Here assumes the camera and experiment field are both immovable and use the mean background model shown in (1).

$$
\begin{aligned}
& \text { Background }(x, y) \\
& =\frac{1}{N} \sum_{i=1}^{N} \operatorname{image}(x, y)
\end{aligned}
$$

The Background $(x, y)$ here is the gray scale value of the point $(x, y)$ on background model image and the image $(x, y)$ is the value on each frame image. Make the summation and averaging to obtain the mean background model. The calculating result is shown below in Figure 6 .

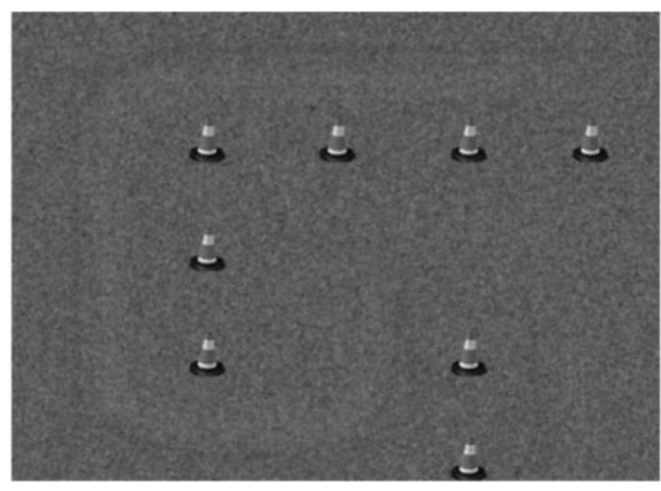

FIGURE VI.BACKGROUND MODEL

Then calculate every difference between each frame image and the background model. The vehicle range can be found after removing the small range of impurities in the larger difference area by filtering. The detection result of the range of the vehicle is shown below in Figure 7 and it shows the feasibility of this method.
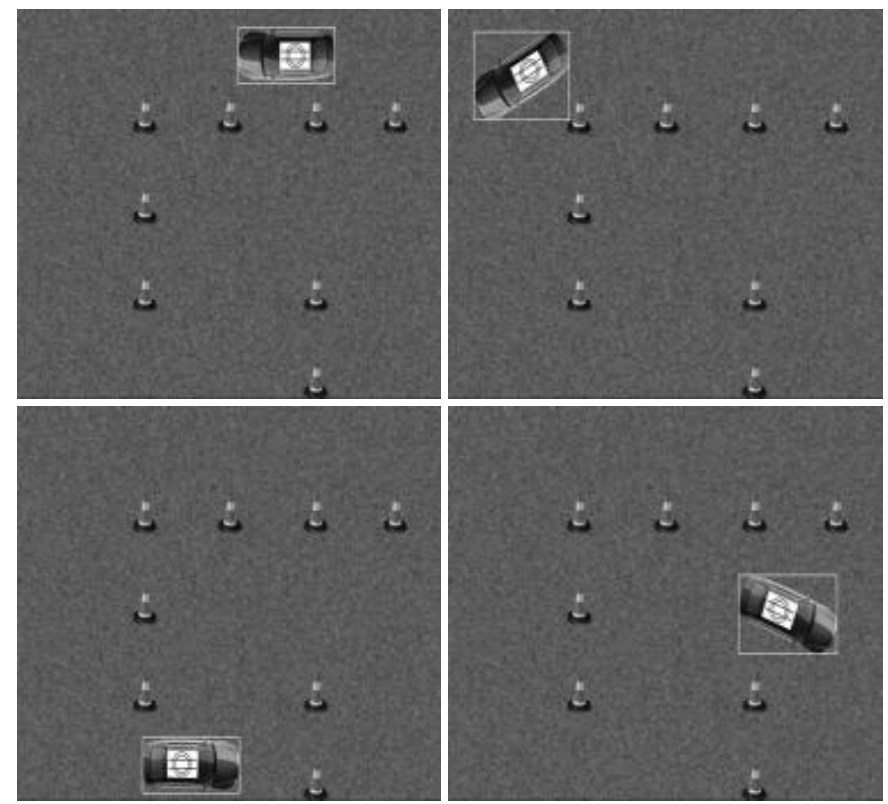

FIGURE VII.DETECTION AREA

Then use Hough transformation to detect the circles and lines in detection area of every single frame, the center of the average circle is the exact position of the vehicle. Mark the positions as solid white points and connect them with a white trajectory line. And the result is shown below in Figure 8.

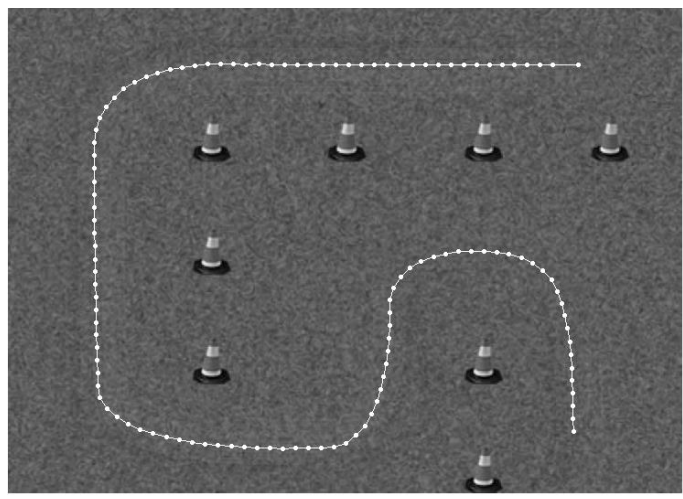

FIGURE VIII. DETECTION RESULT

Analyze the time consumption of this detecting method:

\section{TABLE I.BACKGROUND SUBT RACTION TIME CONSUMPTION}

\begin{tabular}{|c|c|c|c|}
\hline $\begin{array}{c}\text { Background } \\
\text { modeling }\end{array}$ & $\begin{array}{c}\text { Detection area } \\
\text { calculation }\end{array}$ & $\begin{array}{c}\text { Position } \\
\text { detection }\end{array}$ & Total \\
\hline $0.6066 \mathrm{~s}$ & $4.1832 \mathrm{~s}$ & $15.7739 \mathrm{~s}$ & $20.5657 \mathrm{~s}$ \\
\hline
\end{tabular}

According to the data above, calculation for detection area costs $20.3 \%$ of totaltime consumption and it is obvious that the Hough trans formation algorithm costs most of the time which is $76.7 \%$. Speed of processing is about 5.8 FPS (Frames Per Second). To reduce the amount of calculation and the time consumption and to improve the efficiency to satisfy the realtime performance, this paper presents a fast detecting method based on motion prediction. Use the positions witch have been measured accurately in last 3 frames to predict the probable position in current frame. In this way could cut down the time 
consumption of calculation for the differences between every frame and the background model and it reduces the time consumption to a certain extent.

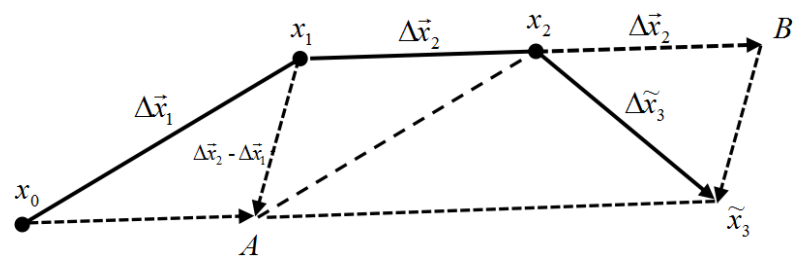

FIGURE IX.PREDICTION PRINCIPLE

Regard the motion of the vehicle as vector form. The $x_{0}, x_{1}, x_{2}$ are positions of the vehicle in last 3 frames, and the $\tilde{x}_{3}$ is the predicted position in current frame. $\Delta \vec{x}_{1}, \Delta \vec{x}_{2}, \Delta \tilde{x}_{3}$ are separately the vector of $x_{0}$ to $x_{1}, x_{1}$ to $x_{2}$ and $x_{2}$ to $\tilde{x}_{3}$. The vehicle moves from $x_{0}$ to $x_{1}$ then $x_{2}$ and finally the position in current frame $x_{3}$. So make the two points $x_{3}$ and $\tilde{x}_{3}$ close as possible.

Due to the continuity of the vehicle movement and high image acquisition frequency in this experiment, the movement will not have significantly drift. So the prediction of $\tilde{x}_{3}$ can be simplified as prediction of $\Delta \tilde{x}_{3}$ by $\Delta \vec{x}_{1}, \Delta \vec{x}_{2}$. Linearly use the difference of $\Delta \vec{x}_{1}$ and $\Delta \vec{x}_{2}$ as the difference of $\Delta \vec{x}_{2}$ and $\Delta \tilde{x}_{3}$ in (2) and use $x_{2}$ and $\Delta \tilde{x}_{3}$ to calculate $\tilde{x}_{3}$ in (3).

$$
\begin{gathered}
\Delta \tilde{x}_{3}-\Delta \vec{x}_{2}=\Delta \vec{x}_{2}-\Delta \vec{x}_{1} \\
\tilde{x}_{3}=x_{2}+\Delta \tilde{x}_{3}
\end{gathered}
$$

Transform vector form into coordinate form. Set $x_{0}\left(r_{0}, c_{0}\right), x_{1}\left(r_{1}, c_{1}\right), x_{2}\left(r_{2}, c_{2}\right), \tilde{x}_{3}\left(r_{3}, c_{3}\right)$ and combine with (2) and (3) to obtain $\tilde{x}_{3}$.

$$
\begin{aligned}
\tilde{x}_{3}\left(r_{3}, c_{3}\right)= & x_{2}\left(r_{2}, c_{2}\right)+2 \Delta \vec{x}_{2}-\Delta \vec{x}_{1} \\
= & x_{2}\left(r_{2}, c_{2}\right)+2\left(x_{2}\left(r_{2}, c_{2}\right)-x_{1}\left(r_{1}, c_{1}\right)\right) \\
& \quad-\left(x_{1}\left(r_{1}, c_{1}\right)-x_{0}\left(r_{0}, c_{0}\right)\right) \\
= & \left(3 r_{2}-3 r_{1}+r_{0}, 3 c_{2}-3 c_{1}+c_{0}\right)
\end{aligned}
$$

When the vehicle is moving, the marker is rotate with the vehicle, and the size of its outer rectangle is not constant so the detection area should be set as dynamic. In the next precise measurement positioning, a new rectangle detection area is formed with point $\tilde{x}_{3}$ as its center and the radius of average circles in last frame multiply $\rho$ as its side. The coefficient $\rho$ is set to 6 according to the size of the marker. The new detection area is shown below in Figure 10 and 11. And then process the detection of the position.

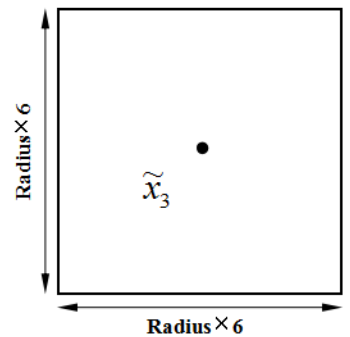

FIGURE X. PREDICTED DETECTINGAREA
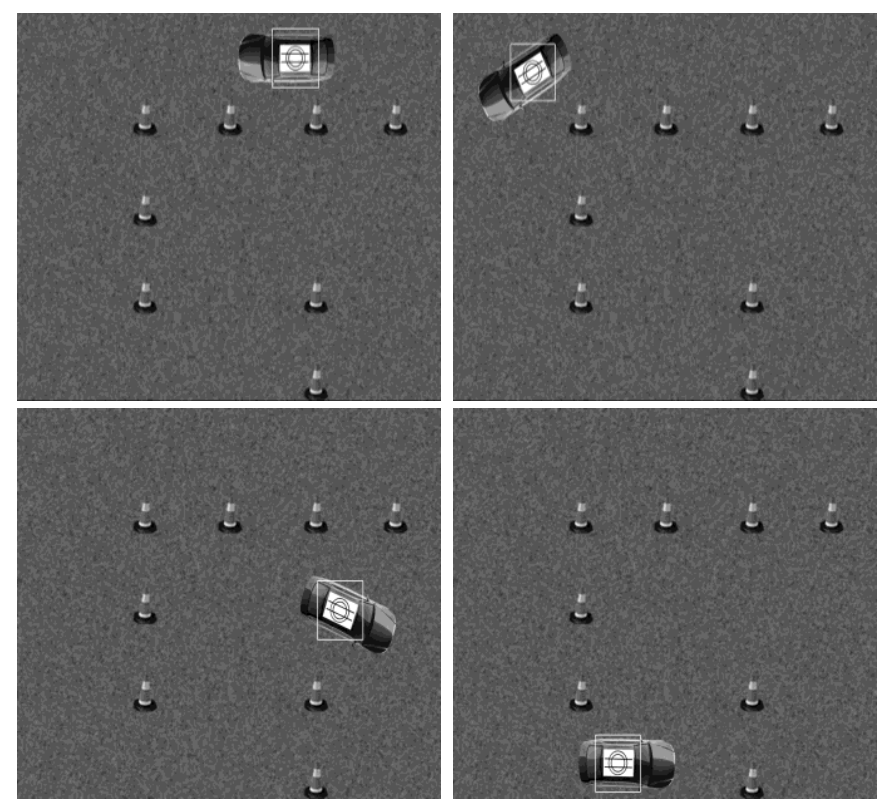

FIGURE XI.DET ECT ION AREA

This method can greatly reduce the detection area which needs Hough transformation, reduce the time consumption, and ensure that the detection area contains the whole marker and accurate detection can be done at the same time. The detection result is shown below in Figure 12.

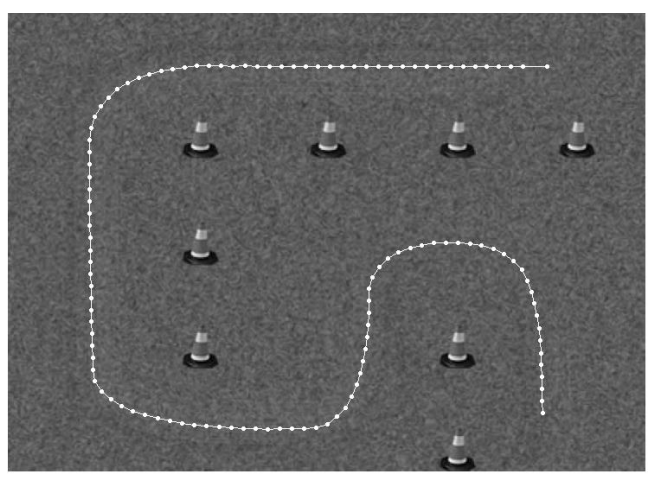

FIGURE XII.DETECT ION RESULT

Also, analyze the time consumption of this method:

TABLE II. MOTION PREDICTION METHOD TIME CONSUMPTION

\begin{tabular}{|c|c|c|c|}
\hline $\begin{array}{c}\text { Background } \\
\text { modeling }\end{array}$ & $\begin{array}{c}\text { Last 3 frames } \\
\text { detection }\end{array}$ & $\begin{array}{c}\text { Position } \\
\text { detection }\end{array}$ & Total \\
\hline $0.6032 \mathrm{~s}$ & $0.3594 \mathrm{~s}$ & $9.6010 \mathrm{~s}$ & $10.5636 \mathrm{~s}$ \\
\hline
\end{tabular}

As is shown above, the detection area calculation is directly replaced by only 3 frames detection and simple predicting calculation of the new detection area which the time consumption can be ignored. At the same time, with the decrease of the size of detection area, precise position detecting algorithm based on Hough transformation costs $39.1 \%$ less than the first method. Together the whole process costs $48.6 \%$ less than the first method. Speed of processing reaches about 11.4 FPS. The real-time performance is satisfied. 


\section{CONCLUSIONS}

This paper analyses the key point of measuring kinematic parameters and summarize the research status of vehicle detection and trajectory tracking and presents a measuring system to measure the key data of kinematic parameters. A simple, practical and fast motion predicting local detection method is presented. The improvement and real-time performance are verified by several experiments. To optimize the system and the detection algorithm, there remains some points to be considered further:

1)Extreme moving situation of the vehicle should be taken in consideration, like running with extreme angular velocity, which might make the marker spin for a big angle and difficult to detect the direction. Or the vehicle meets external conditions that give it extreme change of speed like hitting an obstacle. So the prediction algorithm should be modified and optimized.

2)The experiments as sume that the camera and test field are relative rest and the quality of the frame images obtained from the camera is excellent. But in reality the camera must have a small range of dithering all the time and noise, impurities on them. All of those could cause the problem of detection. So appropriate image filtering algorithm should be studied.

\section{REFERENCES}

[1] Hongiiang Zhang, Yihong Gong. Moving Object Detection,Tracking and Recognition[C]. The 3rd International Conference on Automation, Robotics and Computer Vision, 1994.

[2] Kameda Y, Minoh M. A human motion estimation method using 3 successive video frames[C]// 1996.

[3] Paragios N, Deriche R. Geodesic Active Contours and Level Sets for the Detection and Tracking of Moving Objects[J]. IEEE Transactions on Pattern Analysis \& Machine Intelligence, 2000, 22(3):266-280.

[4] Michalopoulos P G. Vehicle detection video through image processing. the Autoscope system[J]. IEEE Transactions on Vehicular Technology, 2002, 40(1):21-29.

[5] Elgammal A, Harwood D, Davis L. Non-parametric Model for Background Subtraction[C]//European Conference on Computer Vision. Springer-Verlag, 2000:751-767.

[6] Hoose N. IMPACTS: an image analysis tool for motorway surveillance[J]. Traffic Engineering \& Control, 1992, 33(3):140-147.

[7] Abutableb A S. Aut omatic thresholding of gray-level pictures using twodimensional entropy [J]. Computer Vision Graphics \& Image Processing, 1989, 47(1):22-32.

[8] Wixson L. Detecting Salient Motion by Accumulating DirectionallyConsistent Flow[C]// IEEE International Conference on Computer Vision. IEEE, 1999:797-804 vol.2.

[9] Li B, Wang R B, Guo K Y. Study on Machine Vision Based Obstacle Detection and Recognition Model for Intelligent Vehicle[J]. Journal of Highway and Transportation Research and Development, 2002, 19(4):126-129.

[10] Jin D J. Vehicle Detection and Tracking Based on Video[D]. Southwest Jiaotong University, 2008.

[11] Wei W, Gong S F, Gong S C. Improved Video Based Vehicle Detection and Identification Method[J]. Computer Measurement \& Control, 2010, 18(1):20-22.

[12] Zhang Q F, Pu B M, Li T R, Sun H G. Vehicles Detection Based on Histogram of Oriented Gradients and Machine Learning[J]. Computer Systems \& Applications, 2013, 22(7):104-107.

[13] Kang L. Research on Algorithm for Detecting Front Vehicles Based on Convolutional Neural Network[D]. South China University of Technology, 2016.

[14] Mauro E C D, Cootes T F, Taylor C J, Lanitis A. Active Shape Model Search using Pairwise Geometric Histograms[C]// British Machine Vision
Conference 1996, BMVC 1996, University of Edinburgh, Uk. DBLP, 1996.

[15] Setchell C, Dagless E L. Vision-based road-traffic monitoring sensor[J]. IEE Proceedings - Vision Image and Signal Processing, 2001, 148(1):7884.

[16] Kato J, Watanabe T, Joga S, Liu Y, Hase H. An HMM/MRF-based stochastic framework for robust vehicle tracking $[\mathrm{J}]$. IEEE Transactions on Intelligent Transportation Systems, 2004,5(3):142-154.

[17] Badenas J, Sanchiz J M, Pla F. Motion-based segmentation and region tracking in image sequences $\lesssim[J]$. Pattern Recognition, 2001, 34(3):661670 .

[18] Hu W, Xiao X, Xie D, Tan T. Traffic accident prediction using 3-D model-based vehicle tracking[J]. IEEE Transactions on Vehicular Technology, 2004,53(3):677-694.

[19] Ferrier N J, Rowe S M, Blake A. Real-time traffic monitoring[C]// Applications of Computer Vision, 1994. Proceedings of the Second IEEE Workshop on. IEEE, 1994:81-88.

[20] Yu Z, Jain A K, Dubuissonjolly M P. Object Tracking Using Deformable Templates[J]. IEEE Transactions on Pattern Analysis \& Machine Intelligence, 2000, 22(5):544-549.

[21] Tai J C, Tseng S T, Lin C P, Song K T. Real-time image tracking for automatic traffic monitoring and enforcement applications[J]. Image \& Vision Computing, 2004, 22(6):485-501.

[22] Zhang Z, Faugeras O D. Three-dimensional motion computation and object segmentation in a long sequence of stereo frames[J]. International Journal of Computer Vision, 1992, 7(3):211-241.

[23] Coifman B, Beymer D, Mclauchlan P, Malik J. A real-time computer vision system for vehicle tracking and traffic surveillance[J]. Transportation Research Part CEmerging Technologies, 1998, 6(4):271288.

[24] Chen S C, Shyu M L, Zhang C. An intelligent framework for spatiotemporal vehicle tracking[C]// Intelligent Transportation Systems, 2001. Proceedings. IEEE, 2001:213-218.

[25] Tissainayagam P, Suter D. Object tracking in image sequences using point features[J]. Pattern Recognition, 2005, 38(1):105-113.

[26] Li X, Yao X C, Yi L M, Karlsen R, Gerhert G. A Real-Time Vehicle Detection and Tracking System in Outdoor Traffic Scenes.[J]. 2004, 2:761-764.

[27] Sun Z, Miller R, Bebis G, Dimeo D. A Real-time Precrash Vehicle Detection System[C]// IEEE Workshop on Applications of Computer Vision. IEEE Computer Society, 2002:171.

[28] Khammari A, Nashashibi F, Abramson Y, Laurgeau C. Vehicle detection combining gradient analysis and AdaBoost classification[C]// Intelligent Transportation Systems, 2005. Proceedings. IEEE, 2005:66-71.

[29] Zhou X, Huang X Y. Vehicle Following Technique Based on Kalman Filter[J]. Journal of Sichuan Normal University(Natural Science), 2008, 31(6):766-769.

[30] Zhang M H. Visual Target Tracking Algorithm Based on Online Learning[D]. Beijing Jiaotong University, 2012.

[31] Lin L Y. The Study of Vehicle Detection and Tracking Methods Based on Machine Vision[D]. Wuhan University of Science and Technology, 2015.

[32] Wang X H, Liu M Y, Su Y H, Fang L L, Song C M. Video Vehicle Tracking Algorithm Based on Contourlet and MeanShift[J]. Journal of Liaoning Normal University(Natural Science Edition), 2017, 40(2).

[33] Wu G, Zeng X Q, Su S B, Wang C S. Vehicle Appearance Model Tracker Integrating Incremental Principal Component Analysis and Particle Filter[J]. Journal of Nanjing Normal University(Natural Science Edition), 2017, 40(1):33-38.

[34] Zi L, Tao W. A Method for Measuring Kinematic Parameters of Experimental Vehicle Based on Machine Vision[C]// International Conference on Computational Intelligence and Applications. IEEE, 2017:376-381 